\title{
Sorption and Desorption of Phenanthrene onto Iron, Copper, and Silicon Dioxide Nanoparticles
}

\author{
Jing Fang, ${ }^{\dagger}$ Xiao-quan Shan, ${ }^{*},{ }^{\dagger}$ Bei Wen, ${ }^{*},{ }^{\dagger}$ Jin-ming Lin,${ }^{\dagger}$ Xian-cai Lu, ${ }^{*}$ Xian-dong Liu, ${ }^{*}$ \\ and Gary Owens ${ }^{\S}$ \\ State Key Laboratory of Environmental Chemistry and Ecotoxicology, Research Center for \\ Eco-Environmental Sciences, Chinese Academy of Sciences, P.O. Box 2871, Beijing 100085, China, State \\ Key Laboratory of Mineral Deposit Research, Department of Earth Sciences, Nanjing University, \\ Nanjing 210093, China, and Center for Environmental Risk Assessment and Remediation, University of \\ South Australia, Mawson lakes, SA 5095, Australia
}

Received May 12, 2008. Revised Manuscript Received July 11, 2008

\begin{abstract}
The sorption and desorption of phenanthrene by three engineered nanoparticles including nanosize zerovalent iron (NZVI), copper (NZVC), and silicon dioxide $\left(\mathrm{NSiO}_{2}\right)$ were investigated. The sorption of phenanthrene onto $\mathrm{NSiO}_{2}$ was linear and reversible due to the hydrophilic properties of $\mathrm{NSiO}_{2}$. In comparison, sorption of phenanthrene onto NZVI and NZVC was nonlinear and irreversible, which was potentially due to the existence of significantly heterogeneous surface energy distribution patterns detected by a standard molecular probe technique. Naphthalene exerted significant competitive sorption with phenanthrene for NZVI and NZVC, and the isotherm of phenanthrene changed from being significantly nonlinear to nearly linear when naphthalene was simultaneously absorbed. A surface adsorption mechanism was proposed to explain the observed sorption and competition of phenanthrene on both NZVI and NZVC. In contrast, no competition was observed for sorption onto $\mathrm{NSiO}_{2}$. The sorption of phenanthrene on all three nanoparticles significantly decreased with increasing $\mathrm{pH}$. The sorption irreversibility of phenanthrene on NZVI and NZVC were significantly enhanced with decreasing $\mathrm{pH}$. A pH-dependent hydrophobic effect and dipole interactions between the charged surface (electron acceptors) and phenanthrene with electron-rich $\pi$ systems (electron donors) were proposed to explain the observed $\mathrm{pH}$-dependent sorption.
\end{abstract}

\section{Introduction}

Many commercial applications of nanomaterials are currently available including engineered metal nanoparticles for hazardous waste treatment and industry catalysis, silica nanoparticles as solid lubricants and supporting carriers, and carbon nanoparticles in tires. ${ }^{1-6}$ There is no doubt that these engineered nanoparticles will ultimately enter the environment, and their hazardous effects and environmental risks have consequently attracted much attention. Previous studies have demonstrated that engineered nanoparticles have both inflammatory and toxic effects on cells ${ }^{7}$ and that the observed toxicity of the nanoparticles is not only from their own harmful nature but also from the toxic contaminants sorbed by them. ${ }^{8,9}$ In addition, nanoparticulate minerals (including iron oxides), which are widely present in the environment, ${ }^{10}$ have the properties of high surface area, rapid chemical reactivity, and highly dispersed behaviors and exhibit

* Corresponding authors. Tel.: +86-10-62923560. Fax: +86-10-62923563. E-mail:xiaoquan@rcees.ac.cn; bwen@rcees.ac.cn.

${ }^{\dagger}$ Chinese Academy of Sciences.

* Nanjing University.

University of South Australia.

(1) Farrell, J.; Kason, M.; Melitas, N.; Li, T. Environ. Sci. Technol. 2000, 34, 514.

(2) Ponder, S. M.; Darab, J. G.; Mallouk, T. E. Environ. Sci. Technol. 2000, $34,2564$.

(3) Kanel, S. R.; Greneche, J. M.; Choi, H. Environ. Sci. Technol. 2006, 40, 2045.

(4) Chen, Z.; Meng, H.; Xing, G.; Chen, C.; Zhao, Y.; Jia, G.; Wang, T.; Yuan, H.; Ye, C.; Zhao, F.; Chai, Z.; Zhu, C.; Fang, X.; Ma, B.; Wan, L. Toxicol. Lett. 2006, $163,109$.

(5) Vitulli, G.; Bernini, M.; Bertozzi, S.; Pitzalis, E.; Salvadori, P.; Coluccia, S.; Martra, G. Chem. Mater. 2002, 14, 1183.

(6) Grodkowski, J.; Neta, P. J. Phys. Chem. B 2001, 105, 4967.

(7) Peters, K.; Unger, R. E.; Kirkpatrick, C. J.; Gatti, A. M.; Monari, E. J. Mater. Sci. Mater. Med. 2004, 15, 321.

(8) Colvin, V. L. Nat. Biotechnol. 2003, 21, 1160.

(9) Borm, P. J. A. Inhalation Toxicol. 2002, 14, 311 high sorption capacity for organics. Iron nanoparticles are coated with an impermeable barrier of oxide, which can be a model for iron oxide surfaces in minerals, whereas silicon dioxide nanoparticles can represent the silicate mineral surface. Hence, knowledge of toxic compound sorption onto these nanomaterials is critical and helpful for their risk assessment. This knowledge is also useful for understanding the effect of nanomaterials on the fate of toxic compounds in the environment.

Polycyclic aromatic hydrocarbons (PAHs) include a range of distinct compounds that are suspected or known to be potent mutagens and carcinogens that may pose serious human and environmental health risks. PAHs are recognized as aryl hydrocarbon receptor agonists. ${ }^{11}$ Most researchers reported that the sorption of PAHs to solids was mainly due to organic matter present in the solids. ${ }^{12-14}$ However, since the continuing use of engineered nanoparticles is likely to increase, the importance of inorganic solid surfaces interactions with organic contaminants, such as PAHs, will become increasingly important and may become the dominant process and therefore needs to be considered. ${ }^{15-18}$ Whereas Yang and co-workers ${ }^{19,20}$ investigated the sorption and desorption behavior of PAHs to and from carbon

(10) Hochella, M. F.; Lower, S. K.; Maurice, P. A.; Penn, R. L.; Sahai, N.; Sparks, D. L.; Twining, B. S. Science 2008, 319, 1631.

(11) National Recommended Water Quality Criteria; 2002822-R-02-047; Health and Ecological Criteria Division, Office of Science and Technology, U.S. Environmental Protection Agency: Washington, DC, 2002.

(12) Karickhoff, S. W.; Brown, D. S.; Scott, T. A. Water Res. 1979, 13, 241.

(13) Mingelgrin, U.; Gerstl, Z. J. Environ. Qual. 1983, 12, 1.

(14) Chiou, C. T.; Kile, D. E.; Rutherford, D. W.; Sheng, G.; Boyd, S. A. Environ. Sci. Technol. 2000, 34, 1254.

(15) Banerjee, P.; Piwoni, M. D.; Ebeid, K. Chemosphere 1985, 14, 1057.

(16) Huang, W.; Schlautman, M. A.; Weber, W. J., Jr Environ. Sci. Technol. 1996, 30, 2993.

(17) Mader, B. T.; Uwe-Goss, K.; Eisenreich, S. J. Environ. Sci. Technol. 1997, 31, 1079.

(18) Su, Y. H.; Zhu, Y. G.; Sheng, G. Y.; Chiou, C. T. Environ. Sci. Technol. 2006, 40, 6949. 
nanoparticles and found that the adsorption capacity of PAHs on carbon nanomaterials was large and that the sorption isotherms were nonlinear, to the best of our knowledge, there are few studies that have examined the sorption of PAHs onto inorganic nanoparticles.

Therefore, the sorption and desorption behaviors of phenanthrene, a representative PAH, by inorganic nanoparticles was studied in this work. Nanosize zerovalent iron (NZVI), nanosize zerovalent copper (NZVC), and nanosize silicon dioxide $\left(\mathrm{NSiO}_{2}\right)$ were used as three representatives of popular inorganic nanoparticles. The competitive sorption of naphthalene with phenanthrene was examined and the influence of solution $\mathrm{pH}$ on the sorption and desorption of phenanthrene to nanoparticles was also investigated.

\section{Experimental Section}

2.1. Materials and Chemicals. Two high-purity (>99.9\%) engineered metal nanoparticles (NZVI and NZVC) and one nanosize mineral oxide $\left(\mathrm{NSiO}_{2}\right)$ were purchased from Jiechuang Corporation (Guangzhou, China). The average particle sizes of NZVI, NZVC, and $\mathrm{NSiO}_{2}$ were 50,60 , and $20 \mathrm{~nm}$, respectively. The configuration of solid-phase nanoparticles was characterized using TEM and XRD. Nanoparticles surface areas were measured by the $\mathrm{N}_{2}$-BET method (Micrometritics ASAP-2010 volumetric adsorption analyzer, Norcross, GA). To qualitatively characterize the surface energy heterogeneity of nanoparticles, a standard molecular probe technique based on low-temperature nitrogen adsorption was used, and the surface energy distributions of nanoparticles were calculated by a regularization method. ${ }^{21}$ Detailed theories regarding the calculation methods used and the nitrogen adsorption isotherms (Figure S1) are available in the Supporting Information. Phenanthrene and naphthalene were purchased from Sigma-Aldrich Company and used without further purification. All other chemicals were analyticalgrade reagents.

2.2. Batch Experiment for Phenanthrene Sorption and Desorption. Batch sorption studies were conducted in $36 \mathrm{~mL}$ lined capped glass bottles containing $0.01 \mathrm{M} \mathrm{NaNO}_{3}(35 \mathrm{~mL})$ plus 100 $\mathrm{mg} / \mathrm{L} \mathrm{NaN} \mathrm{N}_{3}$ with varying concentrations of phenanthrene on a horizontal motion shaker for $24 \mathrm{~h}$ at $20 \pm 1{ }^{\circ} \mathrm{C}$ (preliminary experiments had shown that all solid-solution mixtures reached apparent equilibrium within $12 \mathrm{~h}$ ). The mass of NZVI, NZVC, and $\mathrm{NSiO}_{2}$ used for the sorption experiments was 200, 200, and $250 \mathrm{mg}$, respectively. Initial concentrations of phenanthrene were controlled to obtain equilibrium concentration ranges over 3 orders of magnitude. The initial $\mathrm{pH}$ values of the batch sorption mixtures were $6.83 \pm$ $0.10,6.87 \pm 0.10$, and $5.84 \pm 0.10$ for $\mathrm{NZVI}, \mathrm{NZVC}$, and $\mathrm{NSiO}_{2}$, respectively. All experiments were conducted in triplicate. Concentrated phenanthrene stock solutions were prepared in methanol and added to the batch vials as needed. Final methanol concentrations in the aqueous phase were less than $0.5 \%$, which has no measurable effect on sorption. ${ }^{22}$ After centrifugation (2000g for $20 \mathrm{~min}$ ) supernatant was sampled and phenanthrene analyzed. For competitive adsorption tests, a fixed dose of naphthalene $(20 \mathrm{mg} / \mathrm{L})$ was added simultaneously with phenanthrene.

For $\mathrm{pH}$ effect tests, the desired initial $\mathrm{pH}$ of NZVI or NZVC or $\mathrm{NSiO}_{2}$ suspensions were adjusted using $0.01 \mathrm{M} \mathrm{NaOH}$ or $0.01 \mathrm{M}$ $\mathrm{HNO}_{3}$, and phenanthrene was added and the batch equilibration initiated immediately. The $\mathrm{pH}$ of the suspension was subsequently re-adjusted twice, at 3.5 and $9.5 \mathrm{~h}$, by dropwise addition of $0.01 \mathrm{M}$ $\mathrm{NaOH}$ or $0.01 \mathrm{M} \mathrm{HNO}_{3}$.

The sorption isotherms were modeled by the Freundlich equation:

$$
Q=K_{\mathrm{f}} C_{\mathrm{e}}^{n}
$$

where $Q(\mathrm{mg} / \mathrm{kg})$ and $C_{\mathrm{e}}(\mathrm{mg} / \mathrm{L})$ are the apparent equilibrium solidphase and solution-phase concentrations, respectively. $K_{\mathrm{f}}[(\mathrm{mg} /$

(19) Yang, K.; Zhu, L. Z.; Xing, B. S. Environ. Sci. Technol. 2006, 40, 1855.

(20) Yang, K.; Xing, B. S. Environ. Pollut. 2007, 145, 529. $\left.\mathrm{kg})(\mathrm{mg} / \mathrm{L})^{-n}\right]$ is the Freundlich affinity coefficient and $n$ is the linearity parameter. Since $K_{\mathrm{f}}$ values are not directly comparable unless $n$ is the same, the concentration-specific solid-water distribution ratio, $K_{\mathrm{d}}(\mathrm{L} / \mathrm{kg})$, is employed: ${ }^{23}$

$$
K_{\mathrm{d}}=Q / C_{\mathrm{e}}
$$

Desorption experiments of phenanthrene were conducted in sequential decant-refill steps immediately following the completion of the sorption experiments. Supernatant $(20 \mathrm{~mL})$ was removed and immediately replaced by the same volume of background solution $\left(0.01 \mathrm{M} \mathrm{NaNO}_{3}+100 \mathrm{mg} / \mathrm{L} \mathrm{NaN}_{3}\right)$ and the vials resealed and shaken for an additional $24 \mathrm{~h}$. After $24 \mathrm{~h}$ of equilibration, the vials were centrifuged, phenanthrene in the supernatant was determined, and the amount of phenanthrene desorbed was calculated from mass differences. The above process was repeated for two complete cycles. Desorption isotherms of phenanthrene under acidic conditions for NZVI $(\mathrm{pH}=3.66)$ and NZVC $(\mathrm{pH}=5.45)$ were tested.

2.3. Determination of Phenanthrene. The concentrations of phenanthrene were determined using a Hewlett-Packard model 1100 gradient HPLC system with a UV-vis detector at an absorption wavelength of $247 \mathrm{~nm}$. The mobile phase was 90:10 methanol:water $(\mathrm{v} / \mathrm{v})$, and the flow rate was $1.0 \mathrm{~mL} / \mathrm{min}$.

Control reactors, prepared identically but containing no sorbent, were simultaneously run to assess loss of solutes, either to the reactor or due to microbial degradation during the sorption test. Results of triplicate sorption at each initial concentration $\left(C_{0}\right)$ showed that the average equilibrium solution-phase concentrations of each solute were consistently within $99-101 \%$ of the respective initial concentration, indicating that microbial degradation, volatilization, or uptake to the glass walls were negligible during the sorption experiments. Previous studies had reported that NZVI could potentially transform benzoic acid to $p$-hydroxybenzoic acid in water. ${ }^{24}$ To ascertain if this was occurring, mass balances were conducted during the sorption studies by determining phenanthrene remaining in nanoparticles in the centrifuge tubes after equilibration and the removal of supernatant. Phenanthrene in nanoparticles was extracted with methanol $(30 \mathrm{~mL})$ and the total recovery was calculated from the sum of the equilibrium amounts in the solution phase and those sorbed in the nanoparticles divided by the initial amount of phenanthrene added. The average recoveries of phenanthrene for NZVI, NZVC, and $\mathrm{NSiO}_{2}$ were $98.5 \%, 100.3 \%$, and $99.2 \%$, respectively, indicating that the phenanthrene degradation during sorption was negligible and, hence, no correction was made for degradation during sorption experiments.

2.4. $\zeta$-Potential Measurements. $\zeta$-potentials of NZVI, NZVC, and $\mathrm{NSiO}_{2}$ at different $\mathrm{pH}$ values were measured in triplicate with a ZetaSizer Nanosize Series instrument (Malvern Instrument Ltd.) using $0.01 \mathrm{M} \mathrm{NaNO}_{3}$ as the background electrolyte.

\section{Results and Discussion}

3.1. Characterization of Nanoparticles. TEM observations showed that both NZVI and NZVC (Figure 1a,b) had very thin layers of oxide shells with distinguishable dark portions inside attributable to $\mathrm{Fe}^{0}$ and $\mathrm{Cu}^{0}$, respectively, whereas $\mathrm{NSiO}_{2}$ aggregates appeared to have no dark portions with an overall texture similar to cotton wool (Figure 1c). Analysis of NZVI and NZVC by XRD detected the three major peaks attributable to $\mathrm{Fe}^{0}$ at $45^{\circ}, 65^{\circ}$, and $83^{\circ}(2 \theta)$ and the three peaks attributable to $\mathrm{Cu}^{0}$ at $43^{\circ}, 50^{\circ}, 75^{\circ}(2 \theta)$ (Figure 2a,b). The weak XRD peak detected for NZVI at $35.3^{\circ}(2 \theta)$ (Figure 2a) indicated a small percentage of iron oxide. Similarly, the weak XRD peaks detected for NZVC between $35^{\circ}$ and $40^{\circ}(2 \theta)$ (Figure $2 \mathrm{~b}$ ) indicated the

(21) Liu, X. D.; Lu, X. C.; Hou, Q. F.; Lu, Z. J.; Yang, K.; Wang, R. C.; Xu, S. J. J. Phys. Chem. B 2005, 109, 15828.

(22) Wang, G. H.; Kleineidam, S.; Grathwohl, P. Environ. Sci. Technol. 2007, $41,1186$.

(23) Pignatello, J. J.; Kwon, S.; Lu, Y. Environ. Sci. Technol. 2006, 40, 7757.

(24) Joo, S. H.; Peitz, A. J.; Sedlak, D. L.; Waite, T. D. Environ. Sci. Technol. 2005, 39, 1263. 

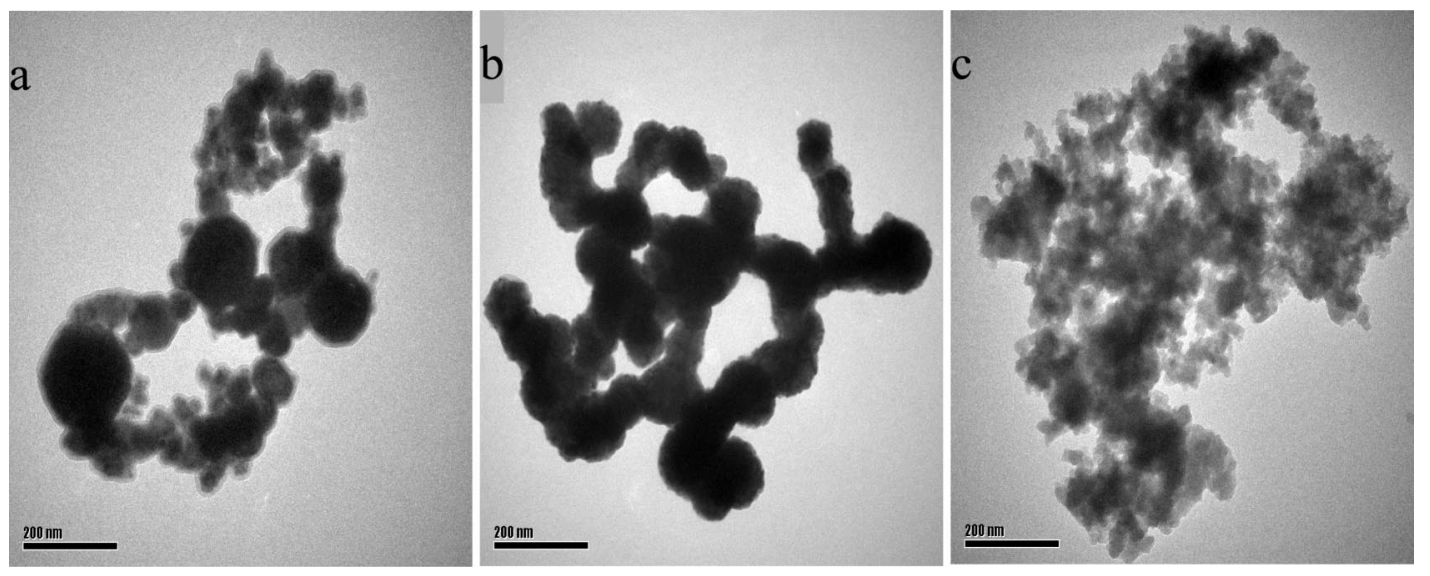

Figure 1. TEM images of (a) NZVI, (b) NZVC, and (c) $\mathrm{NSiO}_{2}$.
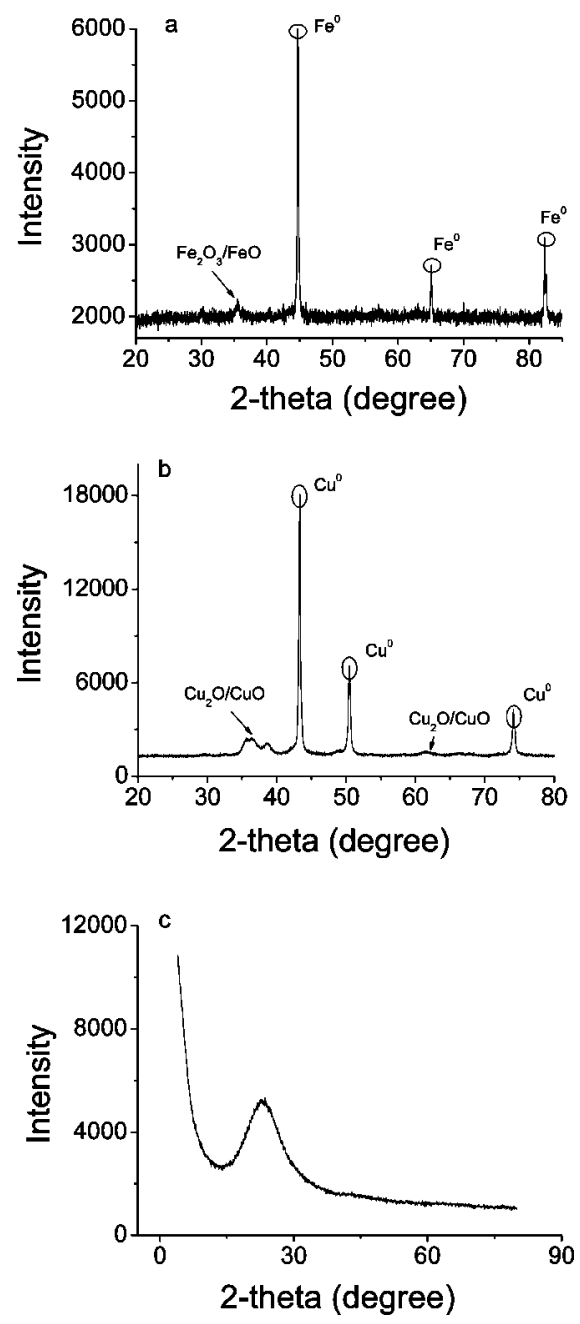

Figure 2. X-ray diffraction analyses of (a) NZVI, (b) NZVC, and (c) $\mathrm{NSiO}_{2}$.

presence of thin layers of copper oxides. The low-intensity wide peak detected in the XRD analysis for $\mathrm{NSiO}_{2}$ (Figure 2c) indicated the presence of an amorphous $\mathrm{SiO}_{2}$ phase. The BET-N 2 surface areas were $11.2,8.7$, and $580.6 \mathrm{~m}^{2} / \mathrm{g}$, and the average pore diameters were 15.1, 17.7, and $4.9 \mathrm{~nm}$ for NZVI, NZVC, and $\mathrm{NSiO}_{2}$, respectively. Since the oxidation of the nanocrystals is a time-dependent process, the residual metals could potentially be an artifact. To determine whether this was the case, the XRD spectra of NZVI and NZVC at the beginning of the present study were compared with those of NZVI and NZVC preserved under vacuum for 6 months. The results demonstrated that the main peaks were almost identical (data not shown), indicating no artifact effects.

Surface energetic heterogeneity was characterized using a molecular probe method. For both NZVI and NZVC there were three peaks (Figure 3a,b). The major peaks were distinguished, suggesting the existence of at least two types of surface energy site zones. There was one low-energy peak at $8.5 \mathrm{~kJ} / \mathrm{mol}$ and two high-energy peaks at 11.5 and $13.5 \mathrm{~kJ} / \mathrm{mol}$ for NZVI, whereas for NZVC, low- and high-energy peaks appeared at 8.5, 12, and $14 \mathrm{~kJ} / \mathrm{mol}$, respectively. The sharp dip in adsorption energy distribution for both NZVI and NZVC at $10 \mathrm{~kJ} / \mathrm{mol}$ was obtained by Langmuir and the Brunauer-Emmett-Teller (BET) local equations, although the peak intensities varied (data not shown). Furthermore, the sharp dip of the bimodal curves did not disappear when changing the $\gamma$ parameter. These results suggested that the surface energies of NZVI and NZVC were significantly heterogeneous. For $\mathrm{NSiO}_{2}$, no distinct two energy peaks existed (Figure 3c), indicating the surface energy was much less heterogeneous. This finding was supported by Heuchel et al. ${ }^{25}$ who used several localized models including Langmuir, BET, the random distribution approximation, the homotattic patch approximation, and mobile adsorption model by Hill and de Boer to evaluate the surface energetic heterogeneity and concluded that the bimodal curves were obtained by Langmuir and BET models, were an essential feature of an adsorbent heterogeneity, and were reproduced by all models.

It should be noted that the surface of the nanoparticles in water is chemically quite different than that experienced in the dry $\mathrm{N}_{2}$ adsorption experiment. However, the heterogeneous surface energy distribution was demonstrated not only by measurement in the dry state with a tiny probe molecule but also by nonlinear sorption of phenanthrene on NZVI and NZVC, detailed in the sorption and desorption paragraph below. The finding of a correlation in sorption behavior (heterogeneous or homogeneous) of two different probe molecules $\left(\mathrm{N}_{2}\right.$ and phenanthrene) on dry and wet surfaces is a significant finding that qualitatively supports the existence of heterogeneous surface energy distribution patterns.

3.2. Sorption and Desorption. Representative sorption and desorption isotherms of phenanthrene for NZVI, NZVC, and $\mathrm{NSiO}_{2}$ are presented in Figure 4. The Freundlich model fitted the experimental sorption data well with correlation coefficients greater than 0.997 (Table 1). The surface area normalized $K_{\mathrm{d}}$

(25) Heuchel, M.; Jaroniec, M.; Gilpin, R. K.; Braeuer, P.; Von Szombathely, M. Langmuir 1993, 9, 2537. 

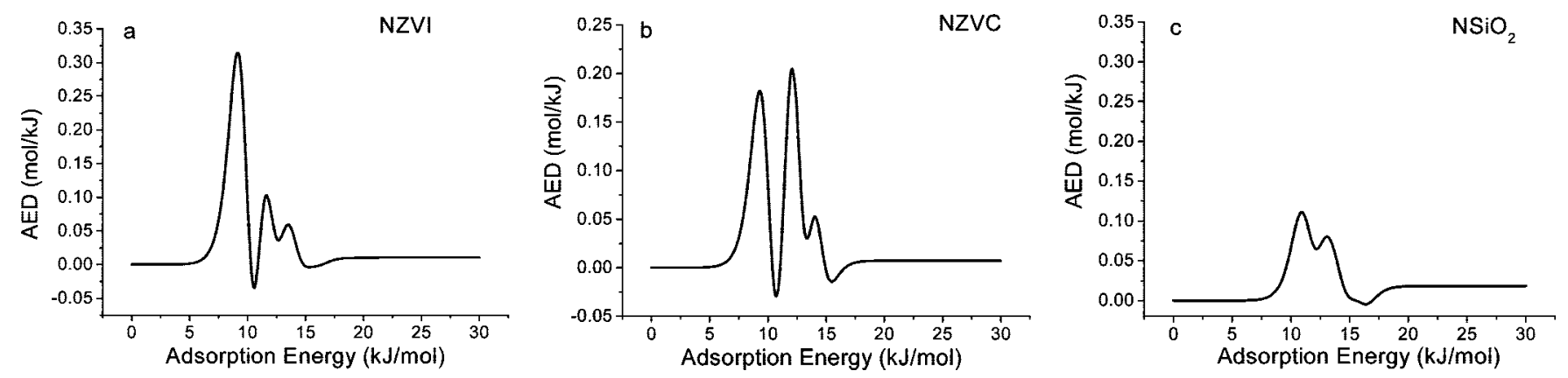

Figure 3. Adsorption energy distribution calculated using the regularization method with $\gamma=0.001$ for (a) $\mathrm{NZVI}$, (b) $\mathrm{NZVC}$, and (c) $\mathrm{NSiO}_{2}$.
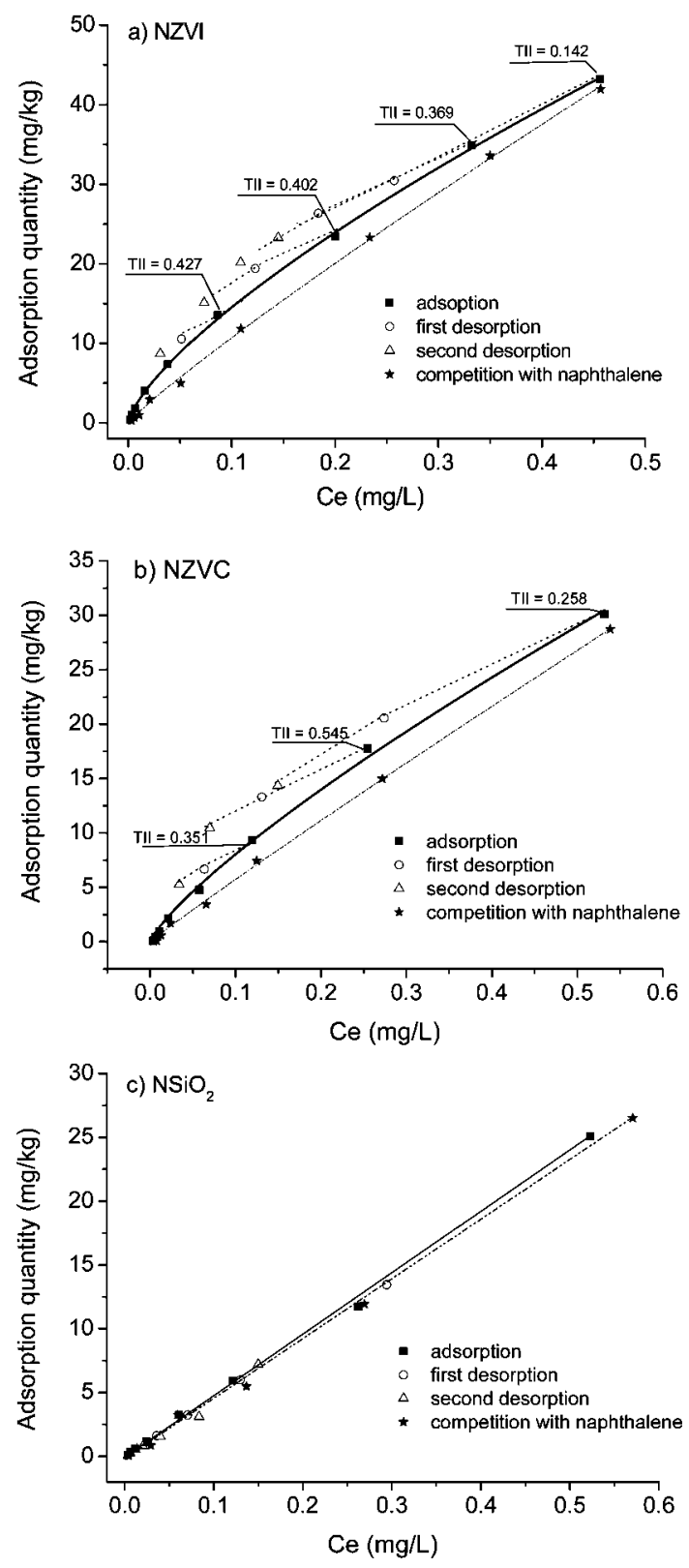

Figure 4. Sorption and desorption isotherms of phenanthrene on (a) NZVI, (b) NZVC, and (c) $\mathrm{NSiO}_{2}$ (the solution $\mathrm{pH}$ is unadjusted: (a) NZVI, $\mathrm{pH}=6.83 \pm 0.10$; (b) $\mathrm{NZVC}, \mathrm{pH}=6.87 \pm 0.10$; (c) $\mathrm{NSiO}_{2}$, $\mathrm{pH}=5.84 \pm 0.10)$ and the sorption isotherms with naphthalene as a competitor were also presented.

values of $\mathrm{NSiO}_{2}$ (the surface area $580.6 \mathrm{~m}^{2} / \mathrm{g}$ ) was $0.069 \mathrm{~mL} / \mathrm{m}^{2}$, which was similar to $0.056 \mathrm{~mL} / \mathrm{m}^{2}$ determined previously for microsize amorphous $\mathrm{SiO}_{2}$ (the surface area $8.23 \mathrm{~m}^{2} / \mathrm{g}$ ). ${ }^{16} \mathrm{Hence}$, the large surface area of $\mathrm{NSiO}_{2}$ was responsible for its relatively higher sorption capacity compared to microsize amorphous $\mathrm{SiO}_{2}$. The sorption affinities $\left(K_{\mathrm{f}}\right)$ were in the order NZVI $>$ NZVC $>$ $\mathrm{NSiO}_{2}$, implying that the type of mineral surface was another important factor controlling the sorption of nonionic hydrophobic organic compounds (HOCs), which was supported by Mader et al. $^{17}$

The $n$ values of the sorption of phenanthrene on NZVI and NZVC were 0.721 and 0.796 , respectively, suggesting nonlinear sorption, which indicated heterogeneous energy distribution of sorption sites on these nanoparticles. In contrast, the sorption of phenanthrene on $\mathrm{NSiO}_{2}$ was linear with an $n$ value of 1.01.

For sorption to hydrophilic mineral surfaces, the interaction energy per unit of surface area of water is reported to be greater than that of HOCs. ${ }^{26}$ Therefore, water would out-compete nonionic HOCs for sorption to mineral surfaces, and the mineral would be coated with at least one layer of strongly sorbed water that prevents the nonionic HOCs from interacting directly with the mineral surface. ${ }^{17,18}$ This hypothesis suggests that the solutes would primarily interact indirectly with the mineral surface and be adsorbed on top of a water film and consequently sorption affinity of nonionic HOCs with a given solid would be largely proportional to the solid surface areas. The sorption of nonionic HOCs to hydrophilic inorganic solids may be mainly caused by London forces and the linear sorption isotherms are believed to be a very unique case observed with hydrophilic minerals (such as $\left.\mathrm{SiO}_{2}\right) .{ }^{18}$ Alternatively, HOCs may simply distribute from the relatively disorganized bulk water to the special volume of ordered water near the solid's surface. ${ }^{26}$ The observed linear sorption for $\mathrm{NSiO}_{2}$ are strongly suggestive that these mechanisms may be in operation for this type of nanoparticle.

Desorption hysteresis is conceptually considered to be a result of pollutant irreversibly bound to sorbents. There are numerous literature references to irreversibility of adsorption. ${ }^{27}$ Rojas et al. $^{28}$ examined the classical and elegant independent sorption theory introduced by Everett from the point of view of the dual site-bond model, a nonindependent pore domain approach that has proven to be very useful for simulating porous networks and capillary phenomena. Desorption hysteresis may be true or due to artificial causes. True hysteresis is referred to as irreversible adsorption and originates from nonreversible processes in which metastable states are produced. Possible artificial hysteresis results from uncontrolled experimental factors such as nonequilibrium owing to intraparticle molecular diffusion limitations or removal

(26) Schwarzenbach, R. P.; Gschwend, P. M.; Imboden, D. M. Environmental Organic Chemistry; Wiley-Interscience: New York, 2003.

(27) Adamson, A. W. Physical Chemistry of Surfaces, 5th ed.; John Wiley \& Sons, Inc. : New York, 1990.

(28) Rojas, F.; Kornhauser, I.; Felipe, C.; Cordero, S. J. Mol. Catal. A: Chem. 2001, 167, 141 . 
Table 1. Freundlich Parameters and $K_{d}$ Values of Sorption Isotherms of Phenanthrene on NZVI, NZVC, and NSiO ${ }_{2}$

\begin{tabular}{|c|c|c|c|c|c|c|c|}
\hline \multirow[b]{2}{*}{ nanoparticles } & \multirow[b]{2}{*}{ solute/cosolute } & \multirow[b]{2}{*}{$K_{\mathrm{f}}$} & \multirow[b]{2}{*}{$n$} & \multirow[b]{2}{*}{$R^{2}$} & \multicolumn{3}{|c|}{$\begin{array}{c}K_{\mathrm{d}}(\mathrm{L} / \mathrm{kg}) \\
\text { initial concentration }(\mu \mathrm{g} / \mathrm{L})\end{array}$} \\
\hline & & & & & 20 & 100 & 800 \\
\hline NZVI & $\mathrm{Phen}^{a} / \mathrm{Naph}^{b}$ & $74.0 \pm 1.3$ & $0.904 \pm 0.02$ & 0.999 & $114 \pm 13$ & $98.3 \pm 1.9$ & $79.7 \pm 3.1$ \\
\hline \multirow[t]{2}{*}{ NZVC } & Phen $^{a}$ & $44.1 \pm 1.1$ & $0.796 \pm 0.02$ & 0.997 & $110 \pm 7$ & $79.1 \pm 4.7$ & $50.2 \pm 1.4$ \\
\hline & $\mathrm{Phen}^{a} / \mathrm{Naph}^{b}$ & $44.4 \pm 0.7$ & $0.958 \pm 0.02$ & 0.999 & $53.3 \pm 5.9$ & $49.8 \pm 4.1$ & $45.6 \pm 1.4$ \\
\hline $\mathrm{NSiO}_{2}$ & $\mathrm{Phen}^{a} / \mathrm{Naph}^{b}$ & $40.2 \pm 0.9$ & $1.05 \pm 0.03$ & 0.998 & $34.2 \pm 0.3$ & $36.8 \pm 1.8$ & $38.9 \pm 0.5$ \\
\hline
\end{tabular}

${ }^{a}$ Phen: phenanthrene. ${ }^{b}$ Naph: naphthalene.

of competing substances, etc. ${ }^{29,30}$ The Thermodynamic Index of Irreversibility (TII) proposed by Sander et al. ${ }^{29}$ can be used to determine the irreversibility of sorption, which is based on the difference in free energy between the real desorption state and the hypothetical fully reversible state. The TII is 0 for completely reversible systems and approaches 1 as the process tends toward complete irreversibility.

To understand the relationship between $\mathrm{pH}$ and the observed desorption hysteresis, we compared the TII values of phenanthrene sorption on NZVI and NZVC at near neutral pH of 6.83 and 6.87 , respectively, with that at acidic $\mathrm{pH}$ of 3.66 and 5.45. The results indicated that under near neutral conditions the TII values were $0.142-0.427$ and $0.258-0.545$ for NZVI and NZVC, respectively (Figure 4a,b), whereas the TII values were $0.804-0.925$ and $0.617-0.956$ for NZVI and NZVC, respectively, under acidic conditions (Figure S2), suggesting more pronounced desorption hysteresis at acidic $\mathrm{pH}$. The reason for more hysteretic desorption at the acidic $\mathrm{pH}$ was discussed in detail in section 3.4.

Under the nearly identical experimental conditions, significant hysteresis during phenanthrene desorption was observed for both NZVI and NZVC but not for $\mathrm{NSiO}_{2}$ (Figure 4c), indicating that the true hysteresis was present for both NZVI and NZVC. At lower initial concentrations of phenanthrene, the TII was generally larger than those observed at higher concentrations of phenanthrene (Figure 4a,b). This was expected since there were only a limited number of high-energy sites available on the solid surface that could be initially occupied. ${ }^{31}$ At lower concentrations of phenanthrene, preferential sorption at the high-energy sites of nanoparticles made sequential desorption more difficult. In contrast, at high concentrations of phenanthrene, the limited highenergy sites became saturated and sorption of phenanthrene occurred at lower energy sites as well, which consequently made the overall desorption easier.

The nonlinear sorption and hysteretic desorption of phenanthrene for both NZVI and NZVC suggested a different mechanism from that of $\mathrm{NSiO}_{2}$. The interaction between an adsorbate and the solid surface of an adsorbent is governed by surface properties, which can in turn be described by the solid surface energetic heterogeneity, which affects the sorption by a direct effect on adsorbate distribution coefficients between solution and nanoparticles surfaces. ${ }^{32}$ Since no in situ method was available to characterize the surface energetic heterogeneity of NZVI, and NZVC in aqueous solution, an ex situ molecular probe method was used and the surface energy distributions of nanoparticles

(29) Sander, M.; Lu, Y.; Pignatello, J. J. J. Environ. Qual. 2005, 34, 1063. (30) Lu, Y. F.; Pignatello, J. J. Environ. Sci. Technol. 2002, 36, 4553.

(31) Wang, X. P.; Shan, X. Q.; Luo, L.; Zhang, S. Z.; Wen, B. J. Agric. Food Chem. 2005, $53,3548$.

(32) Waychunas, G. A.; Kim, C. S.; Banfield, J. F. J. Nanopart. Res. 2005, were calculated by a regularization method. The results suggested that the surface energies of NZVI and NZVC were significantly heterogeneous (Figure 3a,b). We speculate that the observed nonlinear sorptions of phenanthrene on NZVI and NZVC (Figure $4 a, b)$ were potentially due to the significant heterogeneous surface energy distribution patterns to a certain extent, although more confirmatory evidence is needed. This finding was supported by Hou et al. ${ }^{33}$ who demonstrated that the surface energy distribution heterogeneity obtained using dry $\mathrm{N}_{2}$ as a probe gas was related to the adsorption of poly (acrylic acid) at high- and low-energy surface sites of multiwalled carbon nanotubes in aqueous solution.

3.3. Competitive Sorption of Phenanthrene by Naphthalene. The bisolute sorption isotherms of phenanthrene and naphthalene by nanoparticles and their respective Freundlich fitting parameters were presented in Figure 4 and Table 1 . The $n$ values of phenanthrene with naphthalene increased to 0.904 and 0.958 for NZVI and NZVC, respectively, indicating that the sorption nonlinearity of phenanthrene was significantly reduced by the presence of naphthalene. The $K_{\mathrm{d}}$ values of NZVI and NZVC for phenanthrene were also significantly decreased in the presence of naphthalene, which indicated strong competitive sorption between phenanthrene and naphthalene. The degree of competition depended primarily on the phenanthrene concentration; i.e., the competition was more pronounced at lower initial concentrations of phenanthrene $(20$ and $100 \mu \mathrm{g} / \mathrm{L})$ than that at higher initial concentration $(800 \mu \mathrm{g} / \mathrm{L})$. Take NZVI, for example; the $K_{\mathrm{d}}$ values decreased by $58.9 \%$ and $41.4 \%$ at the lower phenanthrene concentrations of 20 and $100 \mu \mathrm{g} / \mathrm{L}$, whereas $K_{\mathrm{d}}$ decreased only by $5.5 \%$ at the higher concentration of $800 \mu \mathrm{g} / \mathrm{L}$. In comparison, no significant differences between $K_{\mathrm{d}}$ values in a mixed naphthalene-phenanthrene system were observed for $\mathrm{NSiO}_{2}$ when compared to the single solute system, indicating no competition with naphthalene.

The significant competitive sorption of naphthalene with phenanthrene by NZVI and NZVC indicated that the solid sorbent surfaces had affinity for both solute molecules at the same sites. In this case, surface adsorption was proposed as the dominant mechanism for sorption of phenanthrene by NZVI and NZVC, whereas sorption is only one monolayer thick for single-solute systems; multilayers could be sorbed in bisolute systems. ${ }^{34}$ The surface adsorption process would be confined to the available surface area. The greater competition of naphthalene for the sorbent when phenanthrene was at relatively low concentration was simply because the high-energy sites were largely occupied by naphthalene, which was present in a large excess concentration (20 mg/L) according to the laws of thermodynamics. However, a solute-coated inorganic surface was possibly more homogeneous

(33) Hou, Q.; Lu, X.; Liu, X.; Hu, B.; Shen, J. J. Colloid Interface Sci. 2004, $278,299$.

(34) Yang, K.; Wang, X. L.; Zhu, L. Z.; Xing, B. S. Environ. Sci. Technol. 2006, 40, 5804 

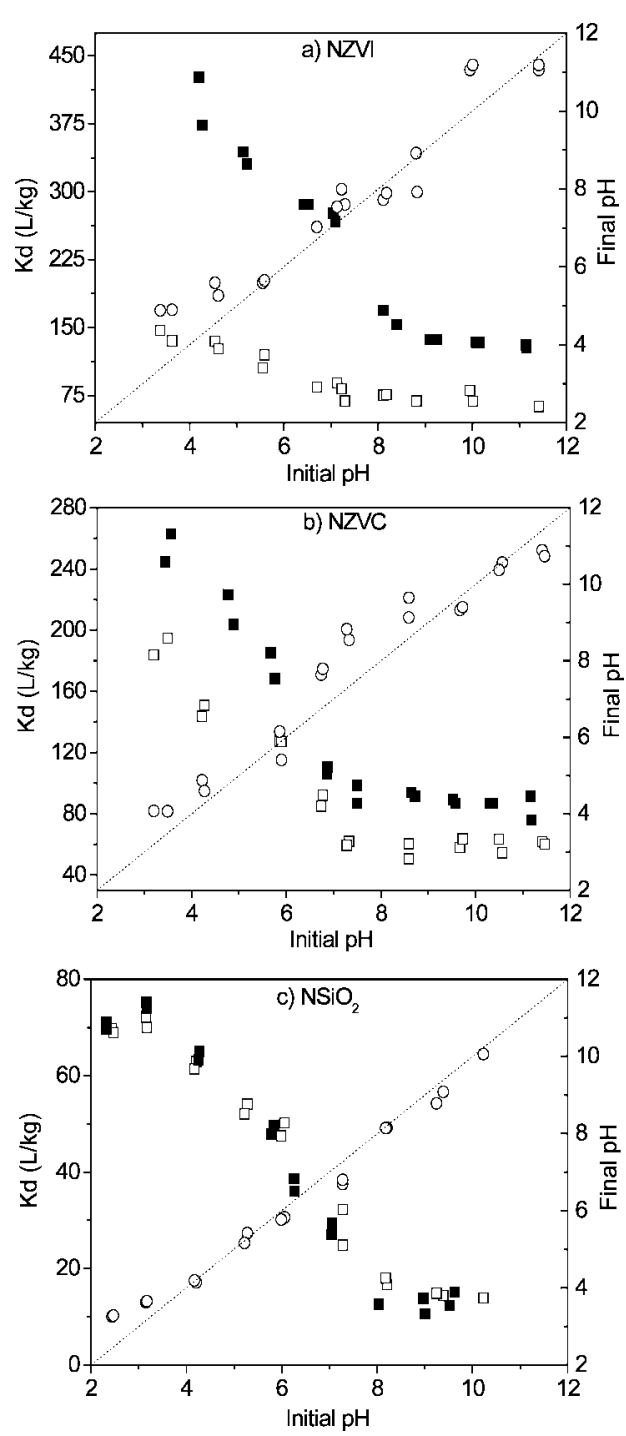

Figure 5. Effect of $\mathrm{pH}$ and initial concentration on sorption coefficients $\left(K_{\mathrm{d}}\right)$ of phenanthrene on nanoparticles [(a) NZVI; (b) NZVC; (c) $\mathrm{NSiO}_{2}$ ]. The solid squares $(\mathbb{\square})$ represent lower initial phenanthrene concentration of $20 \mu \mathrm{g} / \mathrm{L}$ for NZVI, NZVC, and $\mathrm{NSiO}_{2}$. The open squares $(\square)$ represent higher initial phenanthrene concentration of 400,200 , and $200 \mu \mathrm{g} / \mathrm{L}$ for NZVI, NZVC, and $\mathrm{NSiO}_{2}$, respectively. The round open circles (O) represent the final solution $\mathrm{pH}$.

than an uncoated surface, which was mainly responsible for the more linear sorption isotherm of phenanthrene in the presence of competing solute naphthalene.

3.4. Influence of $\mathbf{p H}$ on Phenanthrene Sorption. The nanoparticle-water sorption coefficients $\left(K_{\mathrm{d}}\right)$ of phenanthrene varied with initial $\mathrm{pH}$ and initial phenanthrene concentration (Figure 5). For all three nanoparticles at low and high initial phenanthrene concentrations, the $K_{\mathrm{d}}$ values decreased consistently with increasing $\mathrm{pH}$ under acidic and neutral conditions. However, the $K_{\mathrm{d}}$ values changed a little in alkaline solutions. For both NZVI and NZVC, the $K_{\mathrm{d}}$ values at lower initial concentrations $\left(K_{\mathrm{d}}-\right.$ low $)$ of phenanthrene were much higher than those at higher initial phenanthrene concentrations $\left(K_{\mathrm{d}}\right.$-high), which indicated nonlinear sorption isotherms of phenanthrene in various $\mathrm{pH}$ conditions. In addition, the ratios of $K_{\mathrm{d}}$-low $/ K_{\mathrm{d}}$-high of NZVI and NZVC increased by 1.53 and 1.38 times, respectively, when $\mathrm{pH}$ decreased from 10.1 to 4.2 , indicating enhancement of nonlinear sorption at lower $\mathrm{pH}$, whereas for $\mathrm{NSiO}_{2}$ no significant difference in $K_{\mathrm{d}}$ values was found between the lower and higher initial phenanthrene concentrations at any $\mathrm{pH}$, suggesting that the sorption isotherms were linear at various solution $\mathrm{pH}$ 's. The relationship between the initial and final $\mathrm{pH}$ (Figure 5) shows that the final solutions pHs of $\mathrm{NSiO}_{2}$ were always consistent with the initial $\mathrm{pH}$ except at $\mathrm{pH}$ less than 3 . For $\mathrm{NZVC}$, the final $\mathrm{pHs}$ were a little higher than the initial $\mathrm{pH}$ when initial $\mathrm{pH}$ was less than 9, whereas for NZVI, the final $\mathrm{pHs}$ were $0.65-1.5 \mathrm{pH}$ units higher than the initial $\mathrm{pH}$ when the initial $\mathrm{pH}$ was in the range 3-5. Similar phenomena were also found in previous studies $^{35}$ and were attributed to the continuous protonation and deprotonation of metal nanomaterials in solution.

Previously, changes in solution $\mathrm{pH}$ were found to have no effect on the sorption of phenanthrene by aluminum oxide, iron oxide, and active silica gel. ${ }^{17,18}$ However, Huang et al. ${ }^{16}$ and Stauffer and MacIntyre ${ }^{36}$ showed that phenanthrene sorption on kaolinite, quartz, and goethite was affected by solution $\mathrm{pH}$. They suggested that this $\mathrm{pH}$ effect might be associated with the charge density and the point of zero net charge (PZC) of the sorbent surface, but no further detailed investigations were conducted. To ascertain if the surface charge of the nanomaterials was varying with $\mathrm{pH}$, the electrophoretic mobility ( $\zeta$, zeta potential) of NZVI, $\mathrm{NZVC}$, and $\mathrm{NSiO}_{2}$ at different initial $\mathrm{pHs}$ was measured to detect the surface charge characteristics. The $\zeta$-potential values were described in Figure 6. The PZC of NZVI, NZVC, and $\mathrm{NSiO}_{2}$ were 6.2, 9.2, and 3.6, respectively. Xiao et al. ${ }^{37}$ suggested that when the solution $\mathrm{pH}$ was close to the PZC, the solid surface became less charged and more hydrophobic and consequently favored hydrophobic partition of phenanthrene. This $\mathrm{pH}$ dependent hydrophobic effect could explain the $\mathrm{pH}$-dependent sorption of phenanthrene on $\mathrm{NSiO}_{2}$. However, for NZVI and NZVC, the hydrophobic effect mechanism alone could not explain the $\mathrm{pH}$-dependent sorption of phenanthrene (Figure $5 \mathrm{a}, \mathrm{b}$ ) since the sorption of phenanthrene at $\mathrm{pH}$ less than $\mathrm{PZC}$ did not decrease as expected if this was the only mechanism in operation. Chargeinduced dipole interactions could occur between a charged surface and the electron-rich $\pi$ systems of phenanthrene. ${ }^{17}$ Therefore, the sign and magnitude of surface charge would be important in forming induced dipole interactions between the charged surface and the electron-rich $\pi$ systems. Hence, the alternate mechanism of electro-coupling between phenanthrene (electron donors) and the positive charge (electron acceptors) on the NZVI and NZVC surface was explored to explain the stronger facilitated sorption of phenanthrene at low $\mathrm{pH}$. At lower $\mathrm{pH}$ the fraction of positive charges that may act as an electron acceptor increases and this stronger interaction may not only enhance the nonlinear sorption of phenanthrene on both NZVI and NZVC at lower $\mathrm{pH}$ (Figure 5a,b) but also increase the irreversibility of phenanthrene sorption. This mechanism was supported by the increased TII values of phenanthrene sorption to NZVI and NZVC under an acidic environment (Figure S2). This finding was also supported by Pan et al., ${ }^{38}$ who suggested that the favored $\pi$ electron donor-acceptor interactions in an acidic environment would enhance the binding energy of phenanthrene and thus increase irreversibility.

Changes in $\mathrm{pH}$ may also affect the surface of the nanoparticles materials. However, XRD analyses of nanoparticles at different solution $\mathrm{pH}$ (Figure S3a,c) detected no changes in surface conditions for NZVI and $\mathrm{NSiO}_{2}$ in acid, neutral, and alkaline conditions when compared to their primary XRD spectrum (Figure 2a,c). However, significant copper oxide peaks at $29^{\circ}$ and $36.5^{\circ}$ (2 $\theta)$ appeared in the XRD spectrum of NZVC under acidic

(35) Giasuddin, A. B. M.; Kanel, S. R.; Choi, H. Environ. Sci. Technol. 2007, $41,2022$.

(36) Stauffer, T. B.; MacIntyre, W. G. Environ. Toxicol. Chem. 1986, 5, 949.

(37) Xiao, L.; Qu, X.; Zhu, D. Environ. Sci. Technol. 2007, 41, 2750.

(38) Pan, B.; Ghosh, S.; Xing, B. S. Environ. Sci. Technol. 2007, 41, 6472. 

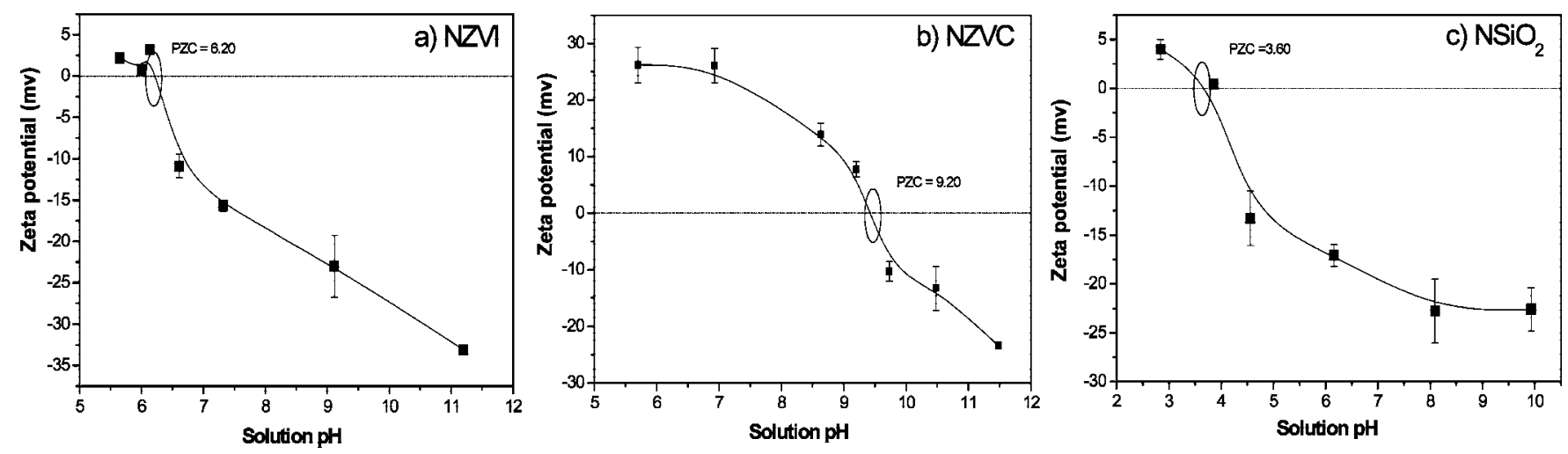

Figure 6. Zeta potentials of (a) NZVI, (b) $\mathrm{NZVC}$ and (c) $\mathrm{NSiO}_{2}$ as a function of solution pH.

conditions $(\mathrm{pH}=3.66)$ (Figure S3b), indicating that for NZVC sorption of phenanthrene by nanoparticles was partially due to the changes in surface configuration induced by $\mathrm{pH}$ changes.

\section{Conclusions}

Batch experiments found that the sorption isotherm of phenanthrene onto $\mathrm{NSiO}_{2}$ was linear and reversible due to the hydrophilic properties of $\mathrm{NSiO}_{2}$, whereas sorption of phenanthrene onto NZVI and NZVC was nonlinear and desorption was hysteretic. Naphthalene exerted significant competitive sorption with phenanthrene for NZVI and NZVC. The linearity parameters ( $n$ values) fitted by the Freundlich model for phenanthrene sorption isotherms increased on NZVI and NZVC in the presence of naphthalene, indicating that the sorption nonlinearity of phenanthrene was significantly reduced by the presence of naphthalene. In contrast, no competition was observed for sorption onto $\mathrm{NSiO}_{2}$. The surface adsorption mechanism was suggested to be the main sorption process of phenanthrene on NZVI and NZVC, and their heterogeneous surface energy distribution patterns were potentially responsible for the sorption. In addition, the sorption of phenanthrene on all three nanoparticles significantly decreased with increasing $\mathrm{pH}$ under acidic and neutral conditions, but changed only a little under alkaline conditions. The desorption hysteresis of phenanthrene from NZVI and NZVC was significantly enhanced under acidic conditions. A pH-dependent hydrophobic effect explained the $\mathrm{pH}$-dependent sorption of phenanthrene on $\mathrm{NSiO}_{2}$, whereas for NZVI and $\mathrm{NZVC}$, dipole interactions between the charged surface (electron acceptors) and phenanthrene with electron-rich $\pi$ systems (electron donors) were also responsible for the observed $\mathrm{pH}$-dependent sorption.

Acknowledgment. This work was funded by the National Natural Science Foundation of China (Grants 20707037 and 40730740).

Supporting Information Available: . Summary of characterization of surface energy heterogeneity, theory of surface energy distributions of NZVI, NZVC, and $\mathrm{NSiO}_{2}$ in aqueous, $\mathrm{N}_{2}$ adsorption isotherms, desorption isotherms of phenanthrene at acidic conditions on NZVI ( $\mathrm{pH}$ $=3.66$ ) and $\mathrm{NZVC}(\mathrm{pH}=5.45)$, and XRD of NZVI, $\mathrm{NZVC}$, and $\mathrm{NSiO}_{2}$ at different solution pHs. This material is available free of charge via the Internet at http://pubs.acs.org.

LA801459S 